\title{
Self-Nanoemulsifying Drug Delivery Systems of Poorly Soluble Drug Dutasteride: Formulation and In-Vitro characterization
}

\author{
Poonguzhali Subramanian, Rajinikant Siddalingam* \\ School of Pharmacy, Taylor's University Lakeside Campus, Kualal Lumpur, Malaysia.
}

\section{ARTICLE INFO \\ Article history: \\ Received on: 30/08/2016 \\ Accepted on: 18/12/2016 \\ Available online: 30/04/2017}

\section{Key words:}

Self emulsifying Drug

Delivery System,

Dutasteride, Poorly soluble

drug, Nanoemulsions,

Enhancement of dissolution.

\begin{abstract}
The present study aims to prepare and evaluate the self-nanoemulsifying drug delivery system (SNEDDS) to enhance the dissolution rate of a poorly soluble drug dutasteride. The formulation was prepared using capryol PGMC, Cremophor EL, and polyethylene glycol (PEG) 400 as oil, surfactant and co-surfactant, respectively. The pseudo-ternary phase diagrams with presence and absence of drug were plotted to find out the nanoemulsification range and also to evaluate the effect of dutasteride on the emulsification behavior of the phases. Prepared SNEDDS formulations were evaluated for its particle size distribution, nanoemulsifying properties, robustness to dilution, self-emulsification time, turbidity measurement, drug content and in-vitro dissolution. Furthermore, the optimized formulations were evaluated for heating cooling cycle, centrifugation studies, freeze thaw cycling, particle size distribution and zeta potential to confirm the stability of the formed SNEDDS formulations. The particle size, zeta potential and polydispersity index of the optimized formulation was found to be $35.45 \mathrm{~nm},-15.45$ and 0.19 , respectively. The in vitro results revealed that the prepared formulation enhanced the dissolution rate of dutasteride significantly compared to pure drug. In 60 minutes, the formulation showed $83.15 \%$ and $82.04 \%$ drug release for $\mathrm{pH} 6.8$ and $\mathrm{pH} 1.2$ respectively. Based on the results, it was concluded that the dutasteride-loaded SNEDDS revealed better dissolution compared to the raw drug suspension and commercial drug.
\end{abstract}

\section{INTRODUCTION}

Benign prostate hyperplasia (BPH) is the most common benign neoplasm which approximately $80 \%$ of the elderly men (above 60 years old) develops the microscopic evidence of BPH (Dolder, 2006). Among them, half of the patients develop enlarged prostate gland, followed by symptoms such as nocturia, increase in urinary urgency and frequency, and reduced force of stream. For patients with enlarged prostate, the treatment would be invasive surgery and prostatectomy. Without surgery, the treatment would be to stop the static factor of prostate gland enlargement, which is dihydrotestosterone (DHT). DHT is a potent androgen, which is converted from testosterone to androstenedione. Inhibition of DHT formation can be a treatment strategy to reduce prostate size. Dutasteride inhibits 5-alpha

* Corresponding Author

Rajinikanth Siddalingam, School of Pharmacy, Taylor's university

Email: rajinikanth.ps @ taylors.edu.my reductase (5AR), which is responsible for the conversion of testosterone to a more potent dihydrotestosterone (Dolder, 2006). Dutasteride is a drug of choice in the group of inhibitors. Compared to other 5AR inhibitor such as finasteride, dutasteride is superior as it binds to both types of alpha-reductases: type I 5AR and type II 5AR. Although type I 5AR is mainly active in skin and liver, and its inhibition, further, reduces the total circulating DHT (Kim, 2013). Dutasteride is 45 times more potent than finasteride to inhibit type 1 5AR, and 2.5 times more potent to inhibit type 2 5AR, thus have more significant effect in reducing DHT count (Keam and Scott, 2008). In a study carried out by Dolder (2006), dutasteride decreases DHT by $~ 95 \%$, as compared to $70 \%$ reduction in patient receiving finasteride based on a four-year observation. It is a recommended regimen for benign prostate hyperplasia treatment to improve symptoms. However, being classified as Biopharmaceutical Classification System (BCS) class II or IV, it has poor aqueous solubility that is only $0.038 \mathrm{ng} / \mathrm{mL}$ in water (Lee et al.,2015) as well as a poor dissolution characteristic (USFDA, 2015). 
This property appears to affect the bioavailability of dutasteride, which slows the dissolution and the problems with absorption are limiting factors. With the marketed oral formulation (Avodart), dutasteride is dissolved in mono-di-glycerides of caprylic acid and capric acid, and then encapsulated in soft-gelatin capsule. However, the achieved bioavailability is still below satisfaction, with only between 40 to 60 per cent (Lee et al., 2015). To achieve optimal therapeutic effects of dutasteride with minimal side effect, dissolution and absorption of dutasteride must be improved. Nowadays, several approaches are available to improve the bioavailability of dutasteride, such as solid dispersion (Kim et al., 2015), polymeric nanoparticle (Choo et al., 2013), Hydroxypropyl- $\beta$ - cyclodextrin (HP- $\beta$-CD) nanostructures (Kim, 2013), and self-emulsifying drug delivery system (SEDDS). SEDDS is a promising method as it is anhydrous isotropic mixture of oil, surfactant, co-surfactant, and drug, which spontaneously form oil-in-water emulsion following the dilution in aqueous medium upon agitation (Abdalla et al., 2008; Balakrishnan et al., 2009; Gursoy and Benita., 2004). Lee et al., (2015), designed supersaturable Self -microemulsifying drug delivery system (SSMEDDS) of dutasteride for oral administration. Ali et al.,( 2014) designed nanoemulsions of dutasteride as transdermal patch. SEDDS formulation is mainly useful for oral formulation where dutasteride can form oil in water (O/W) emulsion in gastrointestinal tract using the GI motility to agitate the mixture (Ammar et al., 2014; Gursoy and Benita., 2004; Tang et al., 2007). Recently, there are many studies has been conducted to improve the bioavailability of the poorly soluble dutasteride. Among them, oral self nanoemulsifying drug delivery system (SNEDDS) of dutasteride has not been researched yet. SNEDDS is selected for formulating because it is simple and cost effective in manufacturing process by omitting high energy emulsification process. SNEDDS is physically a stable lipid solution. It shows a better dissolution rate and expected bioavailability. Hence, the drug dose can be reduced, and brings the adverse effects (Gupta et al., 2011). Khan et al., 2012 developed atorvastatin as SNEDDS and showed $90 \%$ of increased dissolution rate. Zakia et al., 2012 developed SNEDDS of atorvastatin which showed a faster rate (97.24\%) of drug release and 1.87-fold increase in bioavailability. Rajinikanth et al., (2012) investigated on the SNEDDS of valsartan which showed a 6-fold increase in the bioavailability. Balakumar et al., (2013) formulated rosuvastatin as SNEDDS revealed a 2.45 -fold increase in bioavailability. The above studies revealed a significant increase in the bioavailability. The nano sized oil droplet dispersed in gastrointestinal fluid increases the interfacial area; hence it increases its dissolution rate and increases the bioavailability (Amrutkar et al., 2014).

Based on the above findings, the objectives of the study are to develop, to characterize the dutasteride loaded SNEDDS formulation, and to compare the pure drug suspension to the marketed dutasteride.

The strength of marketed dutasteride (Avodart) is $0.5 \mathrm{mg}$ soft gelatin capsule. In this study, the self-nano-emulsifying drug delivery system (SNEDDS) is employed with the strength of 0.5 $\mathrm{mg}$ to enhance the dissolution rate that might increase the bioavailability of dutasteride.

\section{MATERIALS AND METHODS}

\section{Materials}

Dutasteride was kindly offered by RA Chem Pharma (Hyderabad, India), Capryol PGMC, Labrasol, LabrafilM 1966CS, Capryol 90, Cremophor EL, Cremophor 40 RH, Propylene Glycol, Transcutol HP, Solutol HS were obtained as gift samples from Gattefosse (France), sesame oil, and olive oil were obtained from Sigma (USA), Tween 80 was purchased from Merck (Germany) Canola oil was purchased from Lam soon group (Malaysia). PEG 400 and methanol were purchased from SIME Scientific, and hydrochloric acid was purchased from R\&M Chemicals (UK). Methanol was purchased from Merck scientific (Germany), and other solvents used in this study were of analytical grade.

\section{Solubility studies}

The criteria for screening the excipients are the solubility of drug in oils, surfactants and co-surfactants. This screening is performed to determine the solubility of various components and the highest soluble component will be chosen to formulate the SNEDDs. Shake flask method was used to determine equilibrium solubility of dutasteride in various excipients. The solubility of drug in various excipients was determined by adding $2 \mathrm{~mL}$ of each of the selected components to each vial containing an excess of drug. After sealing, the mixture was heated at $40^{\circ} \mathrm{C}$ in a water bath to facilitate the solubilization. Mixing of the systems was performed using a vortex mixer. Formed suspensions were then shaken with a thermodynamically controlled shaker at $30^{\circ} \mathrm{C}$ for 72 hours followed by equilibrium for 24 hours. After reaching equilibrium, each vial was centrifuged at $3000 \mathrm{rpm}$ for 15 minutes, and excess insoluble drug was discarded. Then the supernatant was taken and filtered through the $0.45 \mu \mathrm{m}$ membrane filter. Later, supernatant $10 \mu \mathrm{L}$ oil was taken and suitably diluted with methanol. The concentration of drug was then quantified through RP-HPLC validated method. Methanol $80 \%$ and phosphate buffer pH $6.820 \%$ were used as mobile phase at the wavelength of 230 $\mathrm{nm}$ with the flow rate of $1 \mathrm{~mL} / \mathrm{min}$.

\section{Construction of ternary phase diagrams}

Pseudoternary phase diagrams of oil, surfactant/ cosurfactant $(\mathrm{S} / \mathrm{CoS})$, and water was developed using the water titration method. The mixture of chosen oil and $\mathrm{S} / \mathrm{CoS}$ at certain weight ratio was diluted with water in a drop-wise manner. For each phase diagram at a specific ratio of $\mathrm{S} / \mathrm{CoS}$ (i.e., 1:1,1:2,1:3, $2: 1,3: 1$, and $2: 3$ volume/volume), a transparent and homogenous mixture of oil and S/CoS (Smix) was formed by vortexing for 5 minutes. For each phase diagram a specific Smix ratio was mixed thoroughly in various volume ratios from 0:10 to 10:0. Nine different combinations of oil and Smix (0:10, 1:9, 2:8, 3:7, 4:6, $5: 5,6: 4,7: 3,8: 2,9: 1,10: 0)$ were blended. Each mixture was then titrated with water and visually observed for phase clarity and 
flowability. The concentration of water at which turbidity-totransparency and transparency-to-turbidity transitions occurred was derived. To determine the effect of drug addition on the nanoemulsion boundary, phase diagrams were also constructed in the presence of drug using drug-enriched oil as the hydrophobic component. The physical state of nanoemulsion was marked on a pseudo ternary phase diagram with one axis representing the aqueous phase, second oil and third a mixture of surfactant and cosurfactant at a fixed ratio.

\section{Preparation of SNEDDS}

A series of nanoemulsion formulations was prepared after selecting few of the formulations from the nanoemulsion region of the pseudoternary phase diagram. Accurately weighed drug was placed in a glass vial, oil was added and vortexed until it dissolves, then, surfactant, and finally co-surfactant was added. Then, the components were mixed by gentle stirring, vortex mixing and heating at $40^{\circ} \mathrm{C}$ on a magnetic stirrer, until the drug completely dissolves. The mixture was then stored at room temperature for further study.

\section{Determination of droplet size, polydispersity index and zeta- potential}

One hundred microliters of each formulation was diluted to $10 \mathrm{~mL}$ in a beaker and gently mixed using a magnetic stirrer. Immediately, the average droplet size and zeta potential were determined by dynamic light scattering equipment (Malvern, UK). All studies were done in triplicates.

\section{Determination of Self-emulsification time}

Evaluation of the self-emulsifying properties of formulations were performed by visual assessment. Visual assessment was performed by addition $1 \mathrm{~mL}$ of the pre-concentrate into $\quad 500 \mathrm{~mL}$ of distilled water in dissolution apparatus with a paddle speed of $50 \mathrm{rpm}$. Precipitation was evaluated by visual inspection of the resultant emulsion after 24 hours. The formulations were then categorized as clear (transparent or transparent with bluish tinge), unclear (turbid), stable (no precipitation at the end of 24 hours), or unstable (showing precipitation within 24 hours). The assessment for the efficiency of the emulsion system is also made according to the following grading system

Grade A: Rapidly forming emulsion having a clear or bluish appearance

Grade B: Rapidly forming, translucent bluish appearance

Grade C: Fine milky emulsion forming within 30 seconds

Grade D: Slow forming, slightly oily appearance

\section{Robustness to dilution}

The prepared formulations were diluted infinitely (i.e. 500 times) with distilled water, Phosphate buffer pH 6.80 and acid buffer $\mathrm{pH} 1.2$ in three separate glass vials. The diluted formulations were shaken and then visually inspected after 24 hours for any form of instability.

\section{Determination of viscosity and refractive index}

The viscosity of the SNEDDS formulation was measured by Brookfield viscometer (DV 2T) using at $10 \mathrm{rpm}$. Each reading was taken after equilibrium of the sample at the end of two minutes. The samples were repeated three times. The refractive index of the system was measured using Refractometer (Atago Automatic Digital Refractometer RX-5000, USA) by placing one drop of the formulation on the slide in triplicate at $25^{\circ} \mathrm{C}$.

\section{Thermodynamic stability testing \\ Heating cooling cycle}

Six cycles of cooling and heating between refrigerator temperature $4^{\circ} \mathrm{C}$ and elevated temperature $45^{\circ} \mathrm{C}$ with exposure to each temperature for not less than 48 hours was carried out. The stable formulations were subjected to centrifugation test.

\section{Centrifugation study}

The formulations that pass the heating cooling cycle were centrifuged at $3500 \mathrm{rpm}$ for a period of 30 minutes. The formulations which did not have any phase separation, were considered for the freeze thaw stress test.

\section{Freeze Thawing}

Freeze thawing was employed to evaluate the stability of formulations. The formulations were subjected to 3 to 4 freezethaw cycles, which included freezing at $-4^{\circ} \mathrm{C}$ for 24 hours followed by thawing at $40^{\circ} \mathrm{C}$ for 24 hours. The formulations were observed for phase separation. The stable formulations should not show any phase separation. Those stable formulations which didn't show any phase separation was used for further study.

\section{Attenuated Total Reflectance-Fourier-Transform infrared spectroscopy (ATR-FT-IR) study}

FT -IR spectrum of pure drug, additives and formulation was obtained by FT-IR spectrophotometer. The spectrum obtained from the formulation was compared to the pure drug and marketed product.

\section{Differential Scanning Calorimetry (DSC) study}

DSC curves of the pure drug and the formulation was obtained by DSC endothermic peaks and exothermic peaks. The curves of the formulation were compared to the pure drug and marketed product.

\section{In vitro dissolution study}

Dissolution studies were performed in $900 \mathrm{~mL}$ of buffer pH 1.2 and 6.8 using US Pharmacopeia XXIV dissolution apparatus 2. The formulation was instilled into the apparatus containing $900 \mathrm{~mL}$ of buffer $\mathrm{pH} 1.2$ and 6.8 in a separate run. The temperature was maintained at $37^{\circ} \mathrm{C}$ with the paddle speed of 50 
rpm. Samples $(5 \mathrm{~mL})$ were withdrawn at 5, 15, 30, 45, and 60 minutes with replacement of an equal volume of temperatureequilibrated media. The SNEDDS formulations were used for drug release studies and the results were compared with those of plain drug. The content of the drug in the samples were analyzed $(n=3)$ by using RP-HPLC (Perkin-Elmer).

\section{Accelerated stability testing}

The selected batches of the formulations (F4 and F6) were stored in an incubator at $40^{\circ} \mathrm{C}$ with $75 \%$ of relative humidity for 8 weeks. Visual assessment, droplet size and zeta potential analyses were conducted for selected formulations at the end of the study.

\section{RESULTS AND DISCUSSION}

\section{Solubility studies}

The solubility of dutasteride in various vehicles at room temperature was presented in Fig.1. Components were selected based on two criteria; first, on the solubility profile and second on HLB values. Capryol PGMC is propylene glycol monocaprylate, a water insoluble surfactant but is soluble in many organic solvents, containing more than $90 \%$ of monoester of C8 fatty acid, caprylic acid. It also exerts inhibition to CYP3A4 enzyme, which is useful for drugs which are metabolized by it (Khan et al., 2012). Capryol PGMC has shown highest solubility $\quad(17.35 \mathrm{mg} / \mathrm{mL})$ among the selected oils, thus chosen as oil for preparation of SNEDDS formulation. Cremophor EL (solubility=7.82 $\mathrm{mg} / \mathrm{mL}$ ) and PEG 400 as surfactants and co-surfactant (solubility= $6.85 \mathrm{mg} / \mathrm{mL}$ ) respectively.

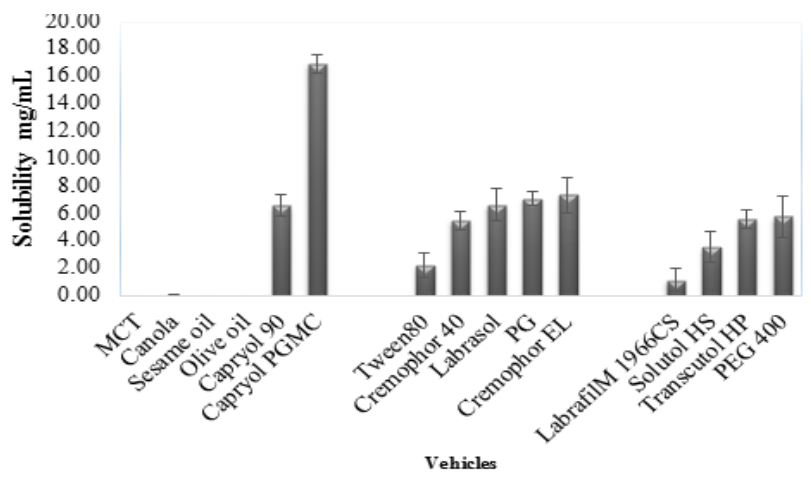

Fig. 1: Saturation solubility of dutasteride in various oils, surfactants and cosurfactants

Cremophor EL, a hydrophilic surfactant with a HLB value of $12-14$, is an ideal surfactant to be used in SNEDDS formulation. According to Weerapol et al., (2014), Cremophor EL has structural significance on the final emulsion droplet size. In their study, they concluded that a branch alkyl structure of Cremophor EL had an effect on the penetration of oil onto the curved film interface, thus resulting in the self-formation of nanoemulsion. Cremophor EL showed highest solubility of drug $(7.82 \mathrm{mg} / \mathrm{mL})$ as compared to other surfactants, so this has been chosen as surfactant for SNEDDS formulation. PEG 400 is a hydrophilic surfactant that is miscible in water, consists of alcohol and ether.

It shows the highest solubility of dutasteride among the co-surfactants and thus it is chosen as co-surfactant. Selection of oil, surfactant and co-surfactant not only based on the solubility, but also on the HLB values. The HLB values for the blend of components was determined by William Griffin's method. Based on William Griffin (1949), the desired HLB value for oil in water emulsion should be in-between 8-18. So based on the HLB values, suitable component was chosen for an $\mathrm{O} / \mathrm{W}$ emulsion.

\section{Construction of pseudo ternary phase diagram}

Based on the results obtained, one of the formulations from the phase diagram was chosen. Oil, surfactant and cosurfactant were grouped into 6 different combinations to study the phase diagrams (Fig.2). Water titration method was used to construct the phase diagram. Pseudo ternary phase diagram was constructed with 5\% to $95 \%$ increment of addition of water. It was also found that by increasing the oil content, the system showed the appearance of coarse emulsion. When co-surfactant is increased in the Smix system, it was observed that there is a decreasing property of spontaneous nanoemulsions. From the observation it is clear that the surfactant is playing an important role in the spontaneous formation of nanoemulsions at appropriate range.

Pseudo ternary phase diagrams were constructed. The samples which were clear or bluish transparent in appearance were chosen as nanoemulsions. From the six different phase diagrams, it was observed that, when Smix ratios increased, nanoemulsions region also increased. The Smix 2:3 resulted in the largest selfnanoemulsifying region than that of other Smix ratios. There was no significant difference in the emulsification region in the $2: 3$ ratios of the oil and surfactant/ co-surfactant with $(0.5 \mathrm{mg})$ and without drug Fig.2.

The droplet size is based on the transparency of the formulation. The more turbid the formulation, droplet size will be bigger and vice-versa. So it is determined that the transparency of the nano-emulsion is inversely proportional to the size of the droplets. At a point, gel was formed with the addition of water, and this will be useful for the gel formulations which can be used as emulsion for external use.

\section{Preparation of Self-nanoemulsifying drug delivery systems (SNEDDS)}

After identifying the emulsification region, the components were selected for further optimization. Capryol PGMC, Cremophor EL and PEG 400 were weighed accurately in the ratio of 2:3 and mixed well. Appropriately weighed dutasteride $0.5 \mathrm{mg}$ was mixed with capryol PGMC until the drug dissolved. Then, the surfactant and co-surfactant were added and mixed thoroughly. Nine points (Table.1) were chosen from the selfnanoemulsifying region and formulation was prepared and was subjected to further studies. 

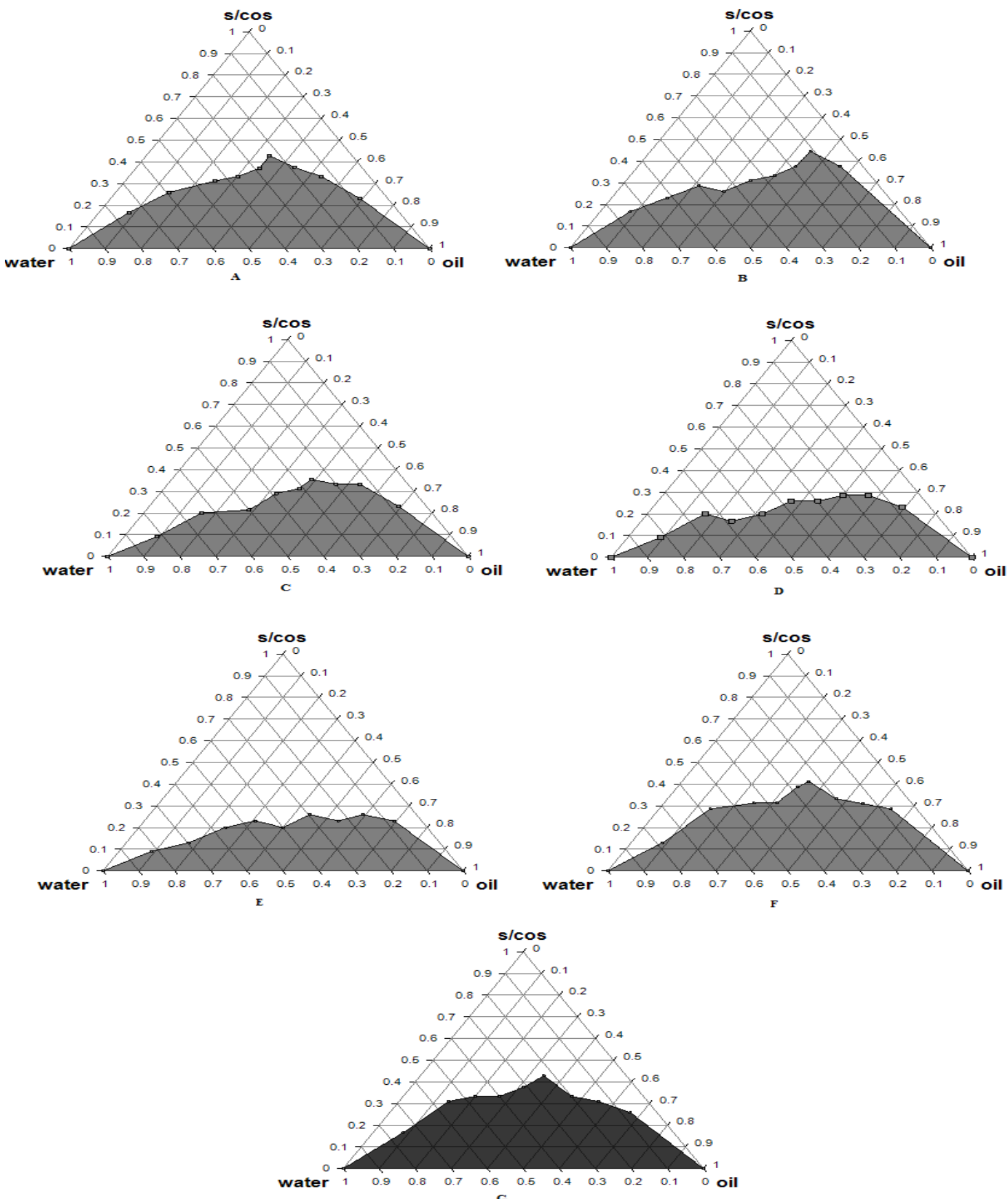

Fig. 2: Pseudoternary phase diagrams of oil (Capryol PGMC), Smix (Cremophor EL and PEG 400) and water with the Smix ratio of A) Smix 1:1; B) Smix 1:2; C) Smix $3: 1 ; \mathbf{D}) \operatorname{Smix} 1: 3 ; \mathbf{E}) \operatorname{Smix} 2: 1 ; \mathbf{F})$ Smix $2: 3 \quad$ G) Smix $2: 3$ with $0.5 \mathrm{mg}$ of dutasteride.

Table 1: Composition each excipient for optimized SNEDDS formulation .

\begin{tabular}{ccccc}
\hline Formulation & Capryol PGMC $(\boldsymbol{\%}$ w/v) & Cremophor EL $(\boldsymbol{\%}$ w/v) & PEG 400 (\% w/v) & Dutasteride (mg) \\
\hline F1 & 20 & 32 & 48 & 0.5 \\
F2 & 30 & 28 & 42 & 18 \\
F3 & 40 & 42 & 36 & 0.5 \\
F4 & 40 & 24 & 25 & 0.5 \\
F5 & 40 & 35 & 30 & 0.5 \\
F6 & 35 & 35 & 35 & 0.5 \\
F7 & 30 & 35 & 32 & 0.5 \\
F8 & 33 & 35 & 25 & 0.5 \\
F9 & 30 & 45 & & 0.5 \\
\hline
\end{tabular}




\section{Determination of droplet size, polydispersity index and zeta- potential}

Determining the particle size is of great importance in formulations as nanoemulsions. Small particle size contributes to greater interfacial area, which then provided better drug partitioning and absorption at GIT. However, there is no consensus in the literature on the exact size range of a nanoformulations. The average particle size and zeta potential of optimized SNEDDS formulation were determined by Photon correlation spectroscopy (PCS) analysis using zeta sizer. The average particle size and zeta potential of nanoemulsions were found to be 45.59 and $11.23 \mathrm{mV}$, respectively (Table 5). PDI measures the size distribution of the nanoparticles in a sample. It has been reported that the mono dispersed samples with uniform particle size distribution has a PDI range from 0.0 to 0.3 , whereas the sample with a broad distribution has a PDI value $>0.4$. In our study, PDI value of all the batches of SNEDDS formulation was found to be between 0.178 to 0.488 . Formulation F1, F2, F4, and F9 indicated uniformity of droplet size suggesting that they were monodispersed with uniform particle size distribution (less than 0.3) as shown in Table 5 and Fig.4. Whereas PDI values for F3, F5, F6, F7, F8 showed non uniformity (more than 0.4 ) of the particles in nanoemulsions formulations. However, ideally accepted, desirable value for PDI is < 0.5 (Rajinikanth et al., 2012; Sunitha et al., 2015). Comparison of particle size with the oils, surfactants, and cosurfactant were presented in the Fig.3. The zeta potential represents the stability of the nanodispersion. It has been reported that nanodispersion with a minimum zeta potential of $<-60 \mathrm{mV}$ has excellent stability, and minimum zeta potential of $<-30 \mathrm{mV}$ has physical stability (Gursoy et al., 2004). All the developed SNEDDS formulation in this study showed zeta potential value, between $-11.23 \mathrm{mV}$ to $-16.86 \mathrm{mV}$ as shown in Table 5 . The small particle size as well as uniform size distribution with optimum zeta potential of developed nanoemulsion was suitable for the development of formulation with enhanced bioavailability (GarcíaDíaz et al., 2012; Yoo et al., 2010).

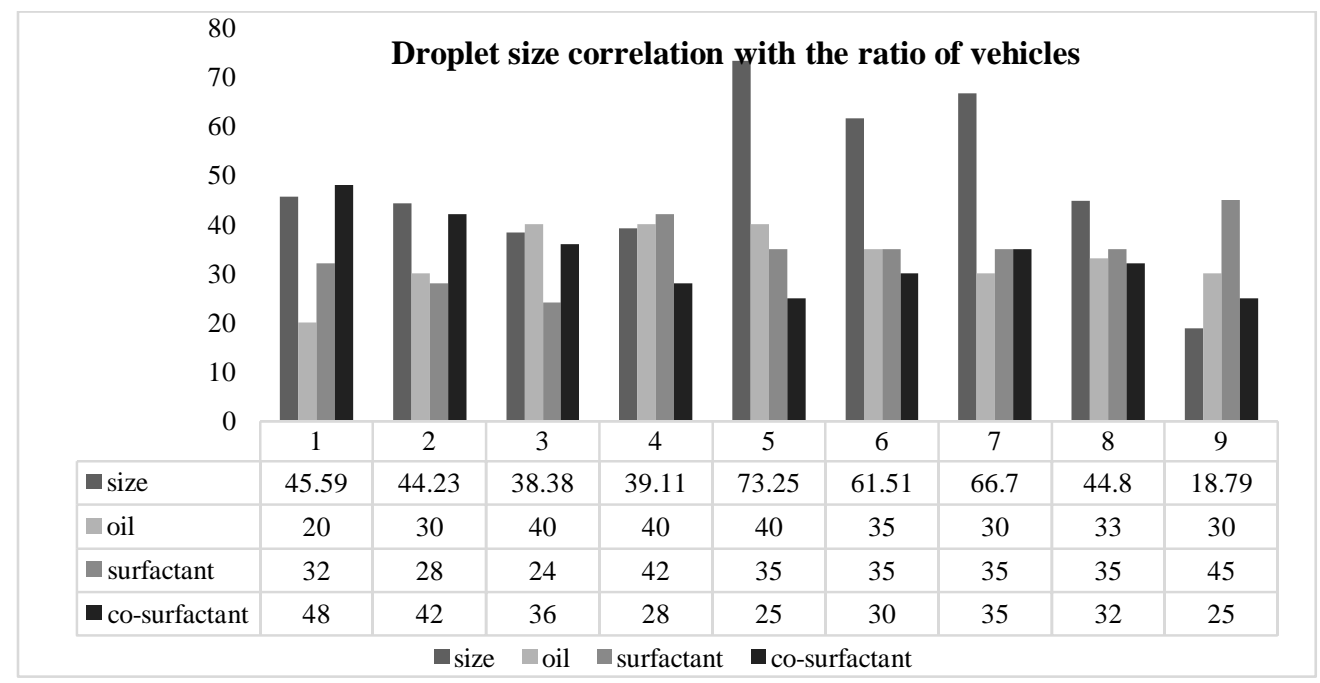

Fig. 3: Particle size (nm) correlation with comparison excipients

Results

$\begin{array}{rlllll} & & & \text { Size (d.n... } & \text { \% Intensity: } & \text { St Dev (d.n... } \\ \text { Z-Average (d.nm): } & 39.11 & \text { Peak 1: } & 42.64 & 96.7 & 16.28 \\ \text { Pdl: } & 0.194 & \text { Peak 2: } & 4299 & 3.3 & 965.8 \\ \text { Intercept: } & 0.932 & \text { Peak 3: } & 0.000 & 0.0 & 0.000 \\ \text { Result quality } & \text { Good } & & & & \end{array}$

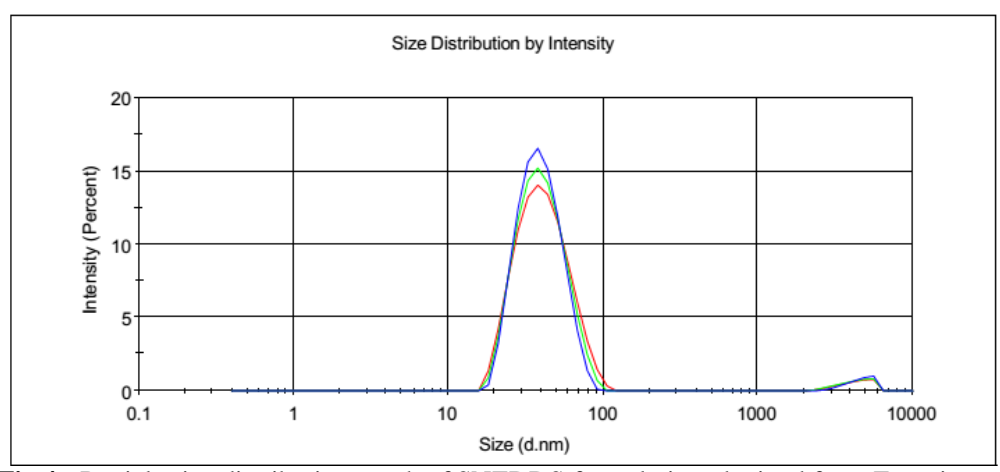

Fig.4: Particle size distribution graph of SNEDDS formulation obtained from Zeta sizer. 


\section{Determination self-emulsification time, viscosity and refractive index}

Most of the prepared SNEDDS formulations formed the nanoemulsion in less than 1 minute with grade $\mathrm{A}$ and $\mathrm{B}$ as shown in Table 3. The formulation F4 and F6 were formed the nanoemulsion within 20 seconds with grade A system in PBS and acid buffer. The results are indicating that the prepared SNEDDS formulations form a good and stable nanoemulsion in different dissolution with short time duration ( $<1$ minute). The viscosity and refractive index were found to be within the range as shown in Table 5.

\section{Thermodynamic stability testing}

It was found (Table.2) that there was no sign of precipitation as well as phase separation for all the formulations except F1 and F8 in heating and cooling cycle, freeze thaw cycle and centrifugation. Hence almost all the SNEDDS formulations passed the thermodynamic stability testing. This reveals that the formulations were robust against the storage of extreme temperatures.

Table 2: Thermodynamic stability testing and self-emulsification parameters of optimized SNEDDS formulation

$\begin{array}{lllll} & & & & \\ & & & & \end{array}$

\section{Robustness to dilution and precipitation}

The dilution capability of the formulations was tested to determine the capability of the formulation to withstand possibly infinite dilutions with water. This was because, upon ingestion, the gastrointestinal fluids are responsible for the dilution, and it is impossible to accurately identify the amount of water present to form emulsion with the formulation Robustness to dilution was performed diluted with excess of water (500-900 mL) and was stored for 12 hours, and the result indicated that there was no precipitation or phase separation as shown in Table 3.

The ability of SNEDDS formulation to withstand aqueous dilution was found to be fascinating. The phenomenon was attributed to the high solubilising properties of the excipients, and also the capability to form a relatively stable emulsion with small droplet sizes. This implied that these formulations were stable at infinite water dilution.
Table 3: The average droplet size of selected SNEDDS formulations at various dilution factors with water.

\begin{tabular}{cccc}
\hline Formulation & $\begin{array}{c}\text { Dilution } \\
\text { factor }\end{array}$ & $\begin{array}{c}\text { Droplet size } \\
(\mathbf{n m})\end{array}$ & PDI \\
\hline F4 & $\mathbf{1 0 0}$ & $\mathbf{5 1 . 3 2} \pm \mathbf{0 . 4 6}$ & $\mathbf{0 . 3 5 2} \pm \mathbf{0 . 0 0 7}$ \\
F4 & $\mathbf{5 0 0}$ & $\mathbf{4 4 . 3 4} \pm \mathbf{0 . 3 2}$ & $\mathbf{0 . 2 0 2} \pm \mathbf{0 . 0 0 3}$ \\
F4 & $\mathbf{9 0 0}$ & $\mathbf{3 8 . 7 6} \pm \mathbf{0 . 9 2}$ & $\mathbf{0 . 1 9 7} \pm \mathbf{0 . 0 0 4}$ \\
F6 & $\mathbf{1 0 0}$ & $\mathbf{1 0 5 . 2 2} \pm \mathbf{0 . 4 1}$ & $\mathbf{0 . 4 7 2} \pm \mathbf{0 . 0 0 2}$ \\
F6 & $\mathbf{5 0 0}$ & $\mathbf{8 2 . 4 9} \pm \mathbf{0 . 3 0}$ & $\mathbf{0 . 4 2 9} \pm \mathbf{0 . 0 0 4}$ \\
F6 & $\mathbf{9 0 0}$ & $\mathbf{6 2 . 2 2} \pm \mathbf{0 . 7 6}$ & $\mathbf{0 . 4 1 4} \pm \mathbf{0 . 0 0 5}$ \\
\hline
\end{tabular}

\section{Effect of droplet size and zeta potential in different dispersant} media

Due to considerable $\mathrm{pH}$ variations along gastrointestinal tracts, it is rational to observe the consequence of different media on the SNEDDS. The optimized SNEDDS formulation was studied in order to find out if there are any changes of particle size and PDI and zeta potential when it is mixed with different dispersion medium such as water, phosphate buffer $\mathrm{pH} 6.8$ and acid buffer $\mathrm{pH}$ 1.2. The results from Table 4 clearly indicated that there were no significant changes in particle size distribution and zeta potential which confirms the stability of the formulation under different $\mathrm{pH}$ conditions.

Table 4: Mean droplet size, PDI and Zeta potential at 500-time dilution with different dispersion medium $(\mathrm{n}=3)$

\begin{tabular}{clccc} 
Pispersion & $\begin{array}{c}\text { Droplet size } \\
\text { Media }\end{array}$ & \multicolumn{1}{c}{ PDI } & & $\begin{array}{c}\text { Zeta } \\
\text { potential } \\
(\mathbf{m V})\end{array}$ \\
\hline F4 & Water & $39.11 \pm 2.32$ & $0.194 \pm 0.005$ & $11.45 \pm 1.42$ \\
F4 & Acid buffer pH1.2 & $41.10 \pm 1.02$ & $0.212 \pm 0.007$ & $8.10 \pm 0.67$ \\
F4 & PBS pH16.8 & $40.61 \pm 0.29$ & $0.201 \pm 0.001$ & $7.95 \pm 0.54$ \\
F6 & Water & $61.51 \pm 0.64$ & $0.410 \pm 0.010$ & $11.67 \pm 0.13$ \\
F6 & Acid buffer pH1.2 & $64.11 \pm 1.10$ & $0.419 \pm 0.002$ & $6.23 \pm 0.87$ \\
F6 & PBS pH16.8 & $63.32 \pm 0.69$ & $0.414 \pm 0.005$ & $7.98 \pm 1.04$ \\
\hline
\end{tabular}

\section{ATR-FT-IR studies}

FT-IR (Spectrum 100, PerkinElmer Life and analytical sciences, USA) was studied for SNEDDS and pure drug to identify any chemical interaction. Compared to the pure drug (Fig.5), it was found that the spectrum of SNEDDS (Fig.6 has same the functional group of dutasteride as follows. Carboxamide, $\mathrm{RCONR}_{2}$. To prove the functional group of amide, there are presence of peaks at N-H stretch (in pure drug $3192.30 \mathrm{~cm}^{-1}$, SNEDDS $3486.01 \mathrm{~cm}^{-1}$ and $\left.3423 \mathrm{~cm}^{-1}\right), \mathrm{N}-\mathrm{H}$ bend $\left(1592.86 \mathrm{~cm}^{-1}\right.$ in pure drug and $1640.23 \mathrm{~cm}^{-1}$ in SNEDDS) and $\mathrm{C}=\mathrm{O}$ stretch at $1670.95 \mathrm{~cm}^{-1}$ in pure drug, whereas $1736.60 \mathrm{~cm}^{-1}$ in SNEDDS. C$\mathrm{C}=\mathrm{C}$, symmetric stretch at the peak of $1670.95 \mathrm{~cm}^{-1}$ was observed in pure drug; and $1640.23 \mathrm{~cm}^{-1}$ in SNEEDS demonstrates the presence of the aromatic ring. As well as the presence of $\mathrm{C}=\mathrm{C}-\mathrm{H}$, asymmetric stretch at $3391.60 \mathrm{~cm}^{-1}$ in pure drug; $3486.01 \mathrm{~cm}^{-1}$ in SNEDDS reveals the presence of aromatic ring. Presence of $\mathrm{C}-\mathrm{X}$ is observed by the peak at $1142.68 \mathrm{~cm}^{-1}$ in the pure drug and $1105.93 \mathrm{~cm}^{-1}$ in SNEDDS. In conclusion, ATR FT-IR spectrum of pure drug and SNEDDS were almost similar because of the presence of the same functional groups. 


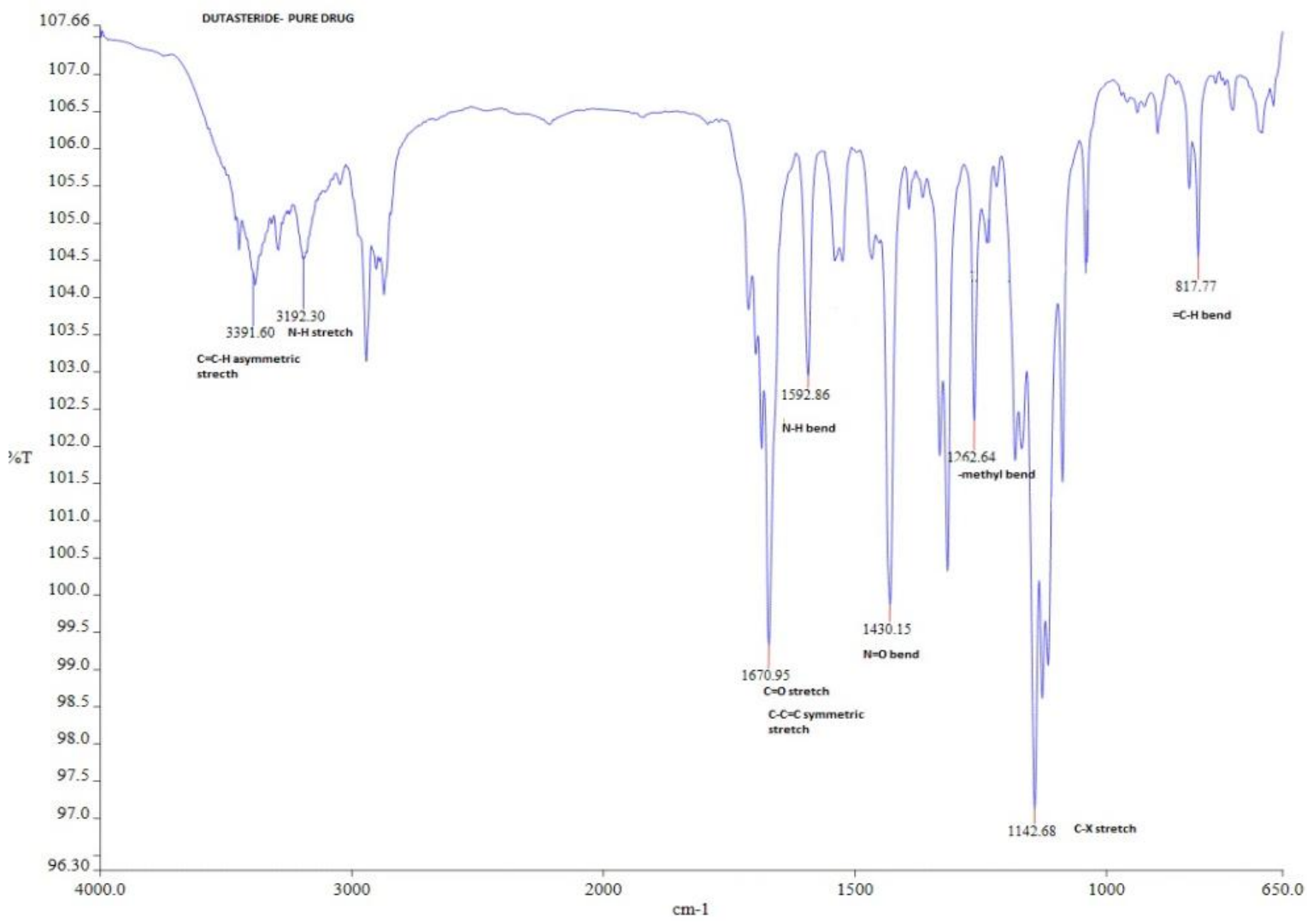

Fig.5: A Chromatogram of FTIR for standard dutasteride.

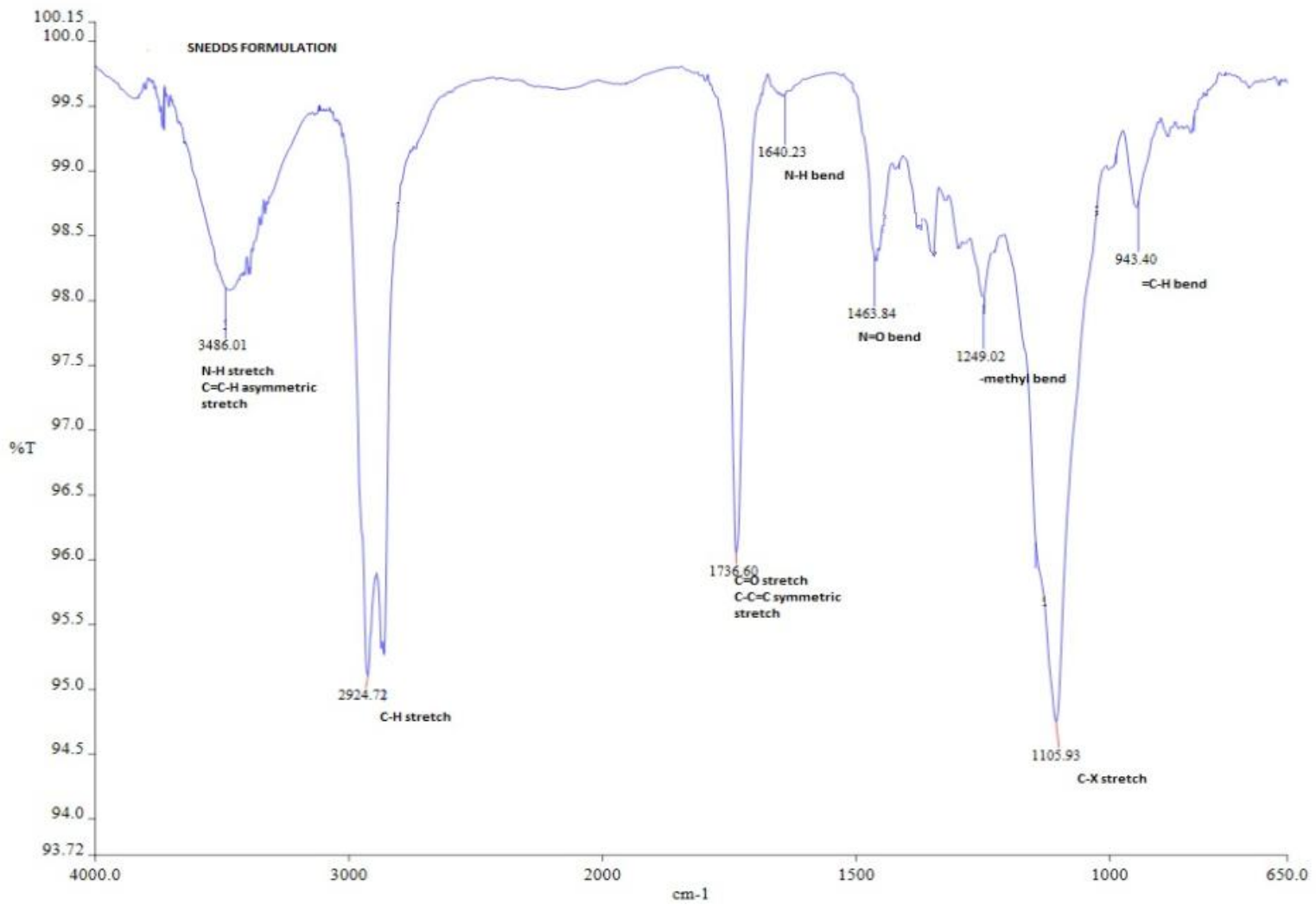

Fig.6: A representative Chromatogram of FTIR for SNEDDS formulation. 


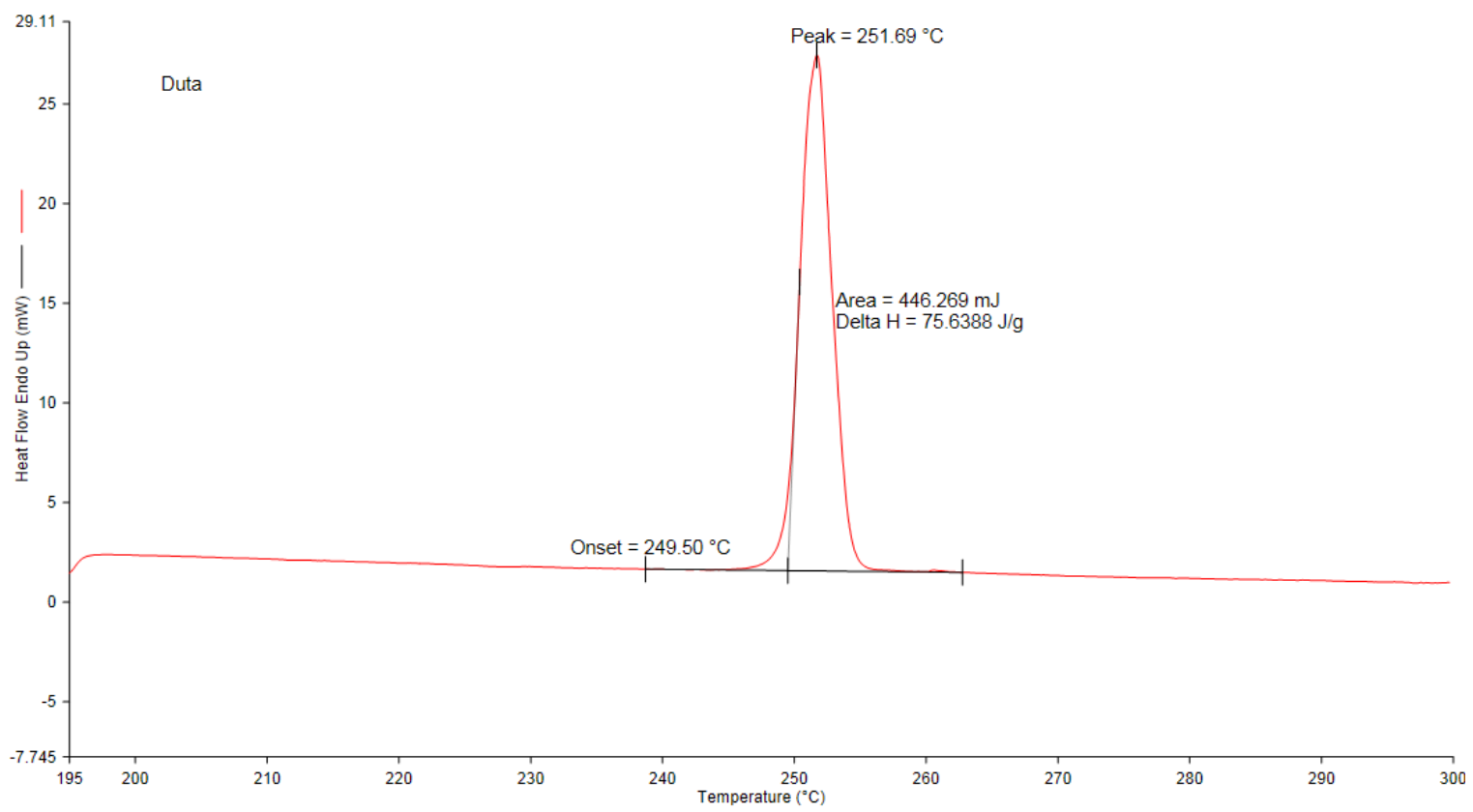

Fig.7: A representative Chromatogram of DSC for standard dutasteride.

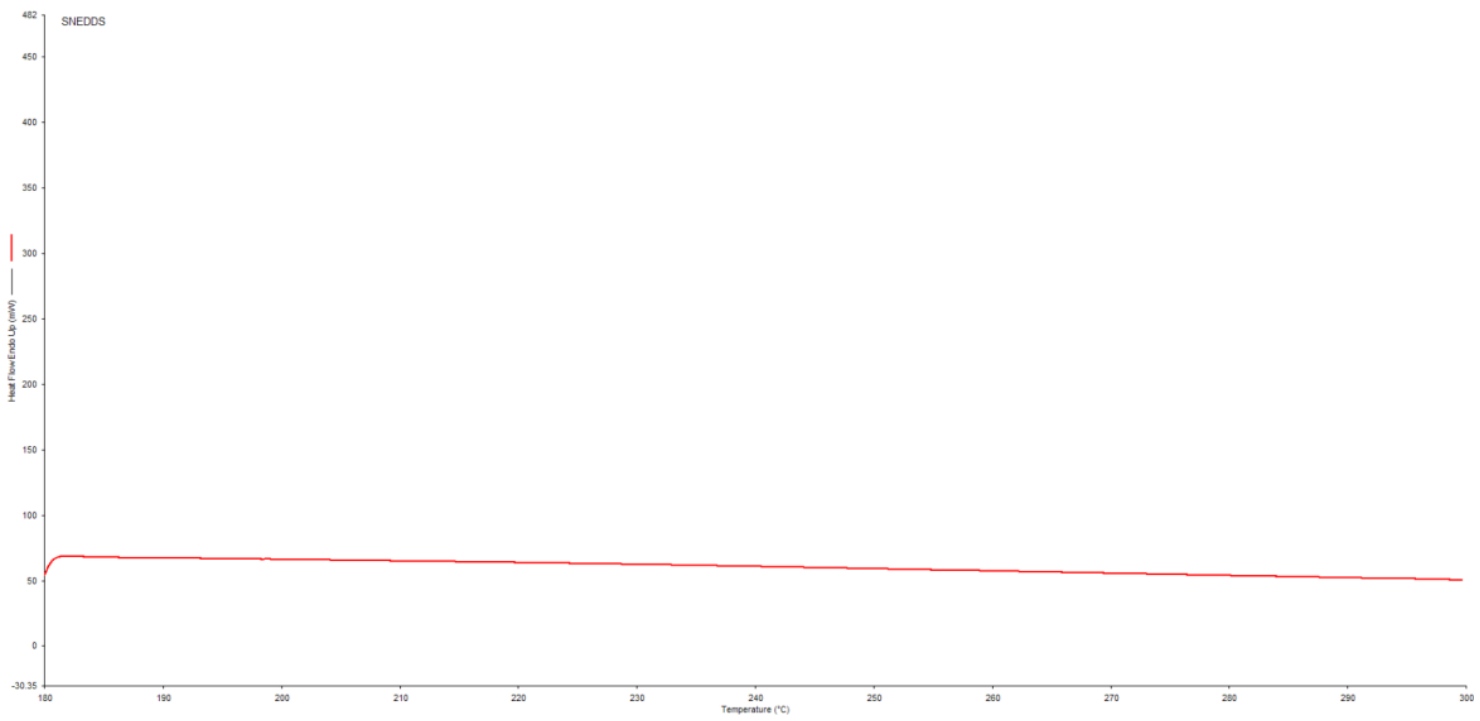

Fig.8: A representative Chromatogram of DSC for SNEDDS formulation.

\section{Differential Scanning Calorimetry (DSC) studies}

DSC (Spectrum 100, PerkinElmer Life and analytical sciences, USA) was used to determine the enthalpy changes by which the trasmission of crystalline form of drug to amorphous form was determined. The melting point of dutasteride is between 242 to $250{ }^{\circ} \mathrm{C}$. The analysis temperature range was chosen between $180-300^{\circ} \mathrm{C}$. Obtained chromatogram is presented in the Fig.7 (pure drug) and Fig. 8 (SNEDDS formulation). It is observed that, there is no curve obtained in the SNEDDS formulation. This could be due to exothermic and endothermic reaction of the drug in the formulation with the excipients. The peak of drug has disappeared in the formulation due to chemical reactions with the other ingredients contained in the formulation. This shows the change of dutasteride. When prepared as SNEDDS formulation, it changed from crystalline to amorphous form. Vadher et al. (2009) studied on the SMEDDS of Neusilin and Ibuprofen also noticed that there was no significant line for the formulation in the chromatogram of DSC studies. They concluded that the drug in the SMEDDS formulation was either present in the amorphous form or in a disordered crystalline form.

\section{In vitro dissolution study}

The in vitro dissolution study of the optimized SNEDDS formulations, raw dutasteride and marketed dutasteride (Avodart) were performed in both acid buffer $\mathrm{pH} 1.2$ and phosphate buffer pH 6.8 and the results were shown in Fig.9 and Fig 10.

Among all the formulations, in pH1.2 (Fig.9) as well as pH6.8 (Fig.10) F4 formulation showed the highest dissolution rate 
in SNEDDS. Based on the Fig.9, there is a significant difference at the $\mathrm{pH} 1.2$, between the raw drug and the SNEDDS formulations. SNEDDS showed better dissolution profile compared to the pure form of the drug at the $\mathrm{pH} 6.8$ (Fig.10). From the observation (Fig.9 and Fig.10), it is understood that the drug release was better in the pH6.8 than $\mathrm{pH} 1.2$. Comparison of the formulations and raw drug indicated that the dissolution profile of the raw drug is worse. This could be due to the poor aqueous solubility. There is no significant $(\mathrm{p} \geq 0.05)$ difference between the $\mathrm{pH} 1.2$ and $\mathrm{pH}$ 6.8, which resembles that the drug is not affected by the $\mathrm{pH}$. F4 showed better drug release at the end of 60minutes than other formulations, commercial product and the raw drug. SNEDDS formulation resulted in better drug release than the raw drug due to relatively low particle size. Rajinikanth et al. (2012) in SNEDDS formulation of valsartan, suggested that the small particle size permitted a faster rate of drug release than the marketed and powder drug. The proper blend of the components, might have played an important role in the globule size reduction, that could have improved the dissolution of the SNEDDS. Balakumar et al. (2013), advised that "the maximum drug release was observed in the proper combination of the components; and also suggested that higher amount of oil, could be the reason for reduced drug release". A better dissolution rate and more predictable bioavailability of SNEDDS indicates that the reduction in drug dose and, perhaps, eliminate the dose-related side effects (Gupta et al., 2011). Therefore, the amount of ingested drug dutasteride could be reduced. Khan et al., (2012) developed selfemulsifying drug delivery system with poorly water-soluble atorvastatin and the results revealed a $90 \%$ of increased dissolution.

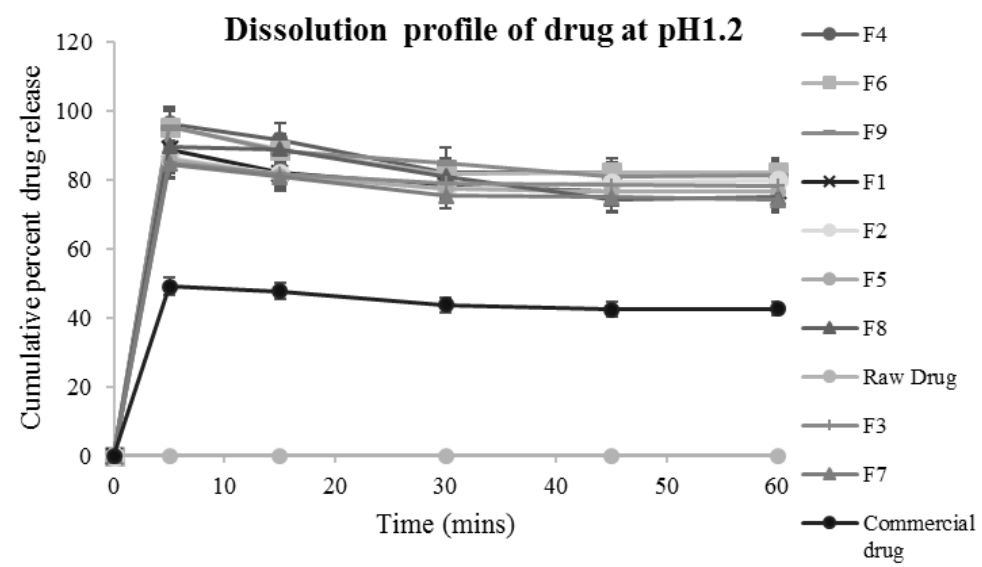

Fig. 9: In vitro dissolution profile of SNEDDS formulation as compared with raw dutasteride using acid buffer pH 1.2.

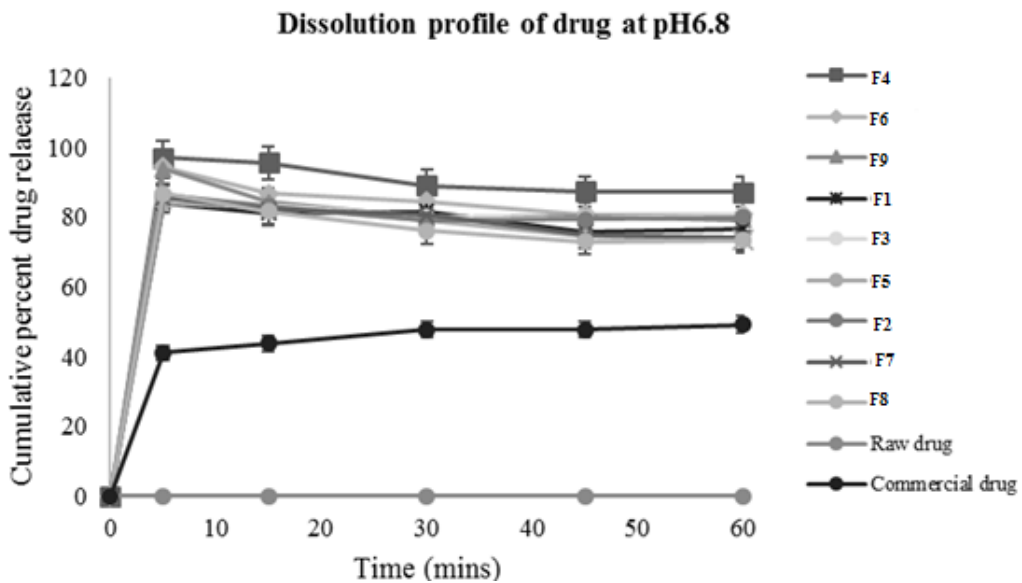

Fig.10: In vitro dissolution profile of SNEDDS formulation as compared with raw dutasteride using PBS buffer $\mathrm{pH}$

Table 5: Droplet size, polydispersity index and zeta-potential.

\begin{tabular}{ccccccc}
\hline Formulation & Droplet size $(\mathbf{n m})$ & PDI & Zeta potential $(\mathbf{m V})$ & Viscosity & Refractive Index & Drug content $(\boldsymbol{\%})$ \\
\hline F1 & $45.59 \pm 0.11$ & $0.185 \pm 0.01$ & $11.23 \pm 0.19$ & $47.4 \pm 1.62$ & $1.443 \pm 0.01$ \\
F2 & $44.23 \pm 0.27$ & $0.178 \pm 0.03$ & $-13.73 \pm 0.03$ & $40.8 \pm 1.64$ & $1.444 \pm 0.02$ \\
F3 & $38.38 \pm 0.36$ & $0.436 \pm 0.03$ & $-14.03 \pm 0.09$ & $44.6 \pm 1.45$ & $1.440 \pm 0.01$ \\
F4 & $39.11 \pm 2.32$ & $0.194 \pm 0.05$ & $15.30 \pm 0.12$ & $40.7 \pm 1.37$ & $1.449 \pm 0.02$ \\
F5 & $73.15 \pm 0.33$ & $0.402 \pm 0.01$ & $15.51 \pm 0.34$ & $40.8 \pm 1.42$ & $1.443 \pm 0.05$ & 99.87 \\
F6 & $61.51 \pm 0.64$ & $0.410 \pm 0.01$ & $-11.67 \pm 0.13$ & $45.4 \pm 1.02$ & $1.440 \pm 0.01$ & 96.84 \\
F7 & $66.70 \pm 1.04$ & $0.415 \pm 0.02$ & $-14.29 \pm 0.21$ & $46.2 \pm 1.38$ & $1.443 \pm 0.02$ & 93.22 \\
F8 & $44.80 \pm 0.16$ & $0.488 \pm 0.04$ & $-16.86 \pm 0.09$ & $40.2 \pm 1.54$ & $1.440 \pm 0.02$ & 92.11 \\
F9 & $48.79 \pm 0.19$ & $0.317 \pm 0.02$ & $-12.71 \pm 0.15$ & $46.8 \pm 1.87$ & $1.441 \pm 0.02$ & 91.74 \\
\hline
\end{tabular}


Table 6: The droplet size, PDI and zeta potential of formulation F4 and F6 before and after accelerated stability study (n=3).

\begin{tabular}{|c|c|c|c|c|c|c|}
\hline \multirow[t]{2}{*}{ Formulation } & \multicolumn{3}{|c|}{ Accelerated stability study (Before) } & \multicolumn{3}{|c|}{ Accelerated stability study (After) } \\
\hline & Droplet size(nm) & PDI & Zeta potential (mV) & Droplet size(nm) & PDI & Zeta potential $(\mathrm{mV})$ \\
\hline F4 & $39.11 \pm 2.32$ & $0.194 \pm 0.005$ & $10.30 \pm 0.12$ & $42.41 \pm 1.24$ & $0.192 \pm 0.07$ & $10.98 \pm 0.10$ \\
\hline F6 & $61.51 \pm 0.64$ & $0.410 \pm 0.010$ & $11.67 \pm 0.13$ & $66.45 \pm 1.43$ & $0.394 \pm 0.02$ & $11.01 \pm 0.16$ \\
\hline
\end{tabular}

\section{Accelerated Stability studies}

The accelerated stability studies were performed at $45^{\circ} \mathrm{C}$ for 3 months. It was observed that there was no phase separation, drug precipitation in the formulations F4 and F6 at the end of 3 months of the accelerated temperature. From the Table 6 , the results revealed that the was no significant changes in the particle size, PDI and zeta potential before and after the accelerated study.

\section{CONCLUSION}

The dutasteride loaded-SNEDDS has been successfully developed using Capryol PGMC, Cremophor EL and PEG 400. The SNEDDS formulations have good droplet size below $100 \mathrm{~nm}$ and pass thermodynamic stability study and robustness to dilution study, confirming the stability of the formulations. The formulations also have the ability to form nanoemulsion less than 1 minute, forming grade $\mathrm{A}$ and grade $\mathrm{B}$ nanoemulsion. The optimized SNEDDs formulations (F2) shows the highest percentage drug release, which is $>85 \%$ at 15 minutes while other formulations show 80-82 \% at 15 minutes. This result draws a big contrast compared to raw drug, whereby negligible amount $(<2$ $\%$ ) dutasteride was detected in the in-vitro dissolution study due to the fact that it is practically insoluble in water. From the study, it is concluded that the SNEDDS formulation is a potentially effective formulation that can be applied to improve the bioavailability of the drug, thereby improve their therapeutic efficacy and minimize the toxicities.

\section{ACKNOWLEDGEMENT}

The current research project was funded by Taylor's University under the TAYLOR'S research grant scheme (TRGS). The code of research grant is, Taylor's university: TRGSMFS/2014/SOP/006.

Conflict of Interests: There are no conflicts of interest.

\section{REFERENCES}

Abdalla A, Klein S, Mäder K. A new self-emulsifying drug delivery system (SEDDS) for poorly soluble drugs: characterization, dissolution, in vitro digestion and incorporation into solid pellets. European journal of pharmaceutical sciences. 2008;35(5):457-64.

Ali MS, Alam MS, Alam N, Siddiqui MR. Preparation, Characterization and Stability Study of Dutasteride Loaded Nanoemulsion for Treatment of Benign Prostatic Hypertrophy. Iranian journal of pharmaceutical research: IJPR. 2014;13(4):1125.

Ammar HO, Ghorab MM, Mostafa DM, Ghoneim AM. Self-nanoemulsifying drug delivery system for sertraline hydrochloride:
Amrutkar C, Salunkhe KS, Chaudhari SR. Review on self nanoemulsifying drug delivery system. American Journal Of Pharmtech Research. 2014;4(3).

Baek IH, Ha ES, Yoo JW, Jung Y, Kim MS. Design of a gelatin microparticle-containing self-microemulsifying formulation for enhanced oral bioavailability of dutasteride. Drug design, development and therapy. 2015; 9:3231.

Balakumar K, Raghavan CV, Abdu S. Self nanoemulsifying drug delivery system (SNEDDS) of rosuvastatin calcium: design, formulation, bioavailability and pharmacokinetic evaluation. Colloids and Surfaces B: Biointerfaces. 2013; 112:337-43.

Balakrishnan P, Lee BJ, Oh DH, Kim JO, Hong MJ, Jee JP, Kim JA, Yoo BK, Woo JS, Yong CS, Choi HG. Enhanced oral bioavailability of dexibuprofen by a novel solid self-emulsifying drug delivery system (SEDDS). European Journal of Pharmaceutics and Biopharmaceutics. 2009;72(3):539-45.

Choo G, Park S, Hwang S and Kim M. Formulation and in vivo Evaluation of a Self-Microemulsifying Drug Delivery System of Dutasteride. Drug Research, 2013; 63(04):203-209.

Date A, Desai N, Dixit $R$ and Nagarsenker M. Selfnanoemulsifying drug delivery systems: formulation insights, applications and advances. Nanomedicine, 2010; 5(10):1595-1616.

Dolder CR. Dutasteride: A dual 5- $\alpha$ reductase inhibitor for the treatment of symptomatic benign prostatic hyperplasia. Annals of Pharmacotherapy. 2006;40(4):658-65.

García-Díaz M., Kawakubo M, Mroz P, Sagristà ML, Mora M, Nonell S \& Hamblin M R. Cellular and vascular effects of the photodynamic agent temocene are modulated by the delivery component. Journal of Controlled Release: Official Journal of the Controlled Release Society, 2012;162(2): 355-63. http://doi.org/10.1016/j.jconrel.2012.07.025 Assessed on 10/08/2016.

Gupta AK, Mishra DK, Mahajan SC. Preparation and in-vitro evaluation of self emulsifying drug delivery system of antihypertensive drug valsartan. Int. J. Pharm. Life Sci. 2011;2(3):633-9.

Gursoy RN, Benita S. Self-emulsifying drug delivery systems (SEDDS) for improved oral delivery of lipophilic drugs. Biomedicine \& Pharmacotherapy. 2004;58(3):173-82.

Keam SJ, Scott LJ. Dutasteride. Drugs. 2008; 68(4):463-85.

Khan F. Systematic Development of Self-Emulsifying Drug Delivery Systems of Atorvastatin with Improved Bioavailability Potential. Scientia Pharmaceutica, 2012; 80(4):1027-1043.

Khan BA, Bakhsh S, Khan H, Mahmood T, Rasul A. Basics of self-micro emulsifying drug delivery system. Journal of Pharmacy and Alternative Medicine, 2012;1(1):13-9.

Kim M. Influence of hydrophilic additives on the supersaturation and bioavailability of dutasteride-loaded hydroxypropyl\&beta; -cyclodextrin nanostructures. International Journal of Nanomedicine, 2013; 2029.

Kim MS, Ha ES, Choo GH, Baek IH. Preparation and in Vivo Evaluation of a Dutasteride-Loaded Solid-Supersaturatable SelfMicroemulsifying Drug Delivery System. International journal of molecular sciences. 2015;16(5):10821-33.

Lee DH, Yeom DW, Song YS, Cho HR, Choi YS, Kang MJ, Choi YW. Improved oral absorption of dutasteride via Soluplus $₫$-based supersaturable self-emulsifying drug delivery system (S-SEDDS). International Journal of Pharmaceutics. 2015; 478(1):341-7.

Rajinikanth PS, Keat NW, Garg S. Self-nanoemulsifying drug delivery systems of Valsartan: preparation and in-vitro characterization. International Journal of Drug Delivery. 2012;4(2):153. 
Sunitha RM, Sowjanya N. Formulation and in-vitro characterization of solid self-nanoemulsifying drug delivery system (sSNEDDS) of simvastatin. Journal of pharmaceutical sciences and research, 2015;7(1): 40-48.

Tang JL, Sun J, He ZG. Self-emulsifying drug delivery systems: strategy for improving oral delivery of poorly soluble drugs. Current drug therapy. 2007;2(1):85-93.

U.S. Food and Drug Administration, U.S. Department of Health and Human Services, viewed November 2015, http://www.accessdata.fda.gov/scripts/cder/dissolution/dsp_SearchResults. cfm.

Vadher AH, Parikh JR, Parikh RH, Solanki AB. Preparation and characterization of co-grinded mixtures of aceclofenac and neusilin US2 for dissolution enhancement of aceclofenac. AAPS PharmSciTech. 2009 ;10(2):606-14.

Weerapol Y, Limmatvapirat S, Nunthanid J, Sriamornsak P. Self-nanoemulsifying drug delivery system of nifedipine: impact of hydrophilic-lipophilic balance and molecular structure of mixed surfactants. AAPS PharmSciTech. 2014;15(2):456-64.

William CG. Classification of surface active agents by "HLB".

SCC Online Journal, 1949; 1(5):311-326. http://journal.scconline.org//pdf/cc1949/cc001n05/p00311-p00326.pdf

Assessed on 15/07/16.

Yoo J H, Shanmugam S, Thapa P, Lee ES, Balakrishnan P, Baskaran R., Yoon S K, Choi HG, Yong CS, Yoo BK, Han K. Novel selfnanoemulsifying drug delivery system for enhanced solubility and dissolution of lutein. Archives of pharmacal research, 2010; 33(3):417426.

Zakia B, Suyang Z, Wenli Z, Junlin W. Formulation Development and Bioavailability Evaluation of a Self -Nanoemulsifying Drug Delivery System (SNEDDS) of Atorvastatin Calcium. International Journal of Pharmaceutics, 2012; 29 (1): 1103-1113.

\section{How to cite this article:}

Siddalingam R, Subramaniam P. Self-Nanoemulsifying Drug Delivery Systems of Poorly Soluble Drug Dutasteride: Formulation and In-Vitro characterization. J App Pharm Sci, 2017; 7 (04): 011022. 